\title{
Transformation Rules for Platform Independent Testing: An Empirical Study
}

\author{
Anders Eriksson \\ Informatics Research Center \\ University of Skövde, Sweden \\ Email: anders.eriksson@his.se \\ Saab Aeronautics, Linköping \\ Email: anders.k.eriksson@saabgroup.com
}

\author{
Birgitta Lindström \\ Informatics Research Center \\ University of Skövde, Sweden \\ Email: birgitta.lindstrom@his.se
}

\author{
Jeff Offutt \\ Software Engineering \\ George Mason University \\ Fairfax, VA 22030-4444 USA \\ Email: offutt@gmu.edu
}

\begin{abstract}
Most Model-Driven Development projects focus on model-level functional testing. However, our recent study found an average of $67 \%$ additional logic-based test requirements from the code compared to the design model. The fact that full coverage at the design model level does not guarantee full coverage at the code level indicates that there are semantic behaviors in the model that model-based tests might miss, e.g., conditional behaviors that are not explicitly expressed as predicates and therefore not tested by logic-based coverage criteria.

Avionics standards require that the structure of safety critical software is covered according to logic-based coverage criteria, including MCDC for the highest safety level. However, the standards also require that each test must be derived from the requirements. This combination makes designing tests hard, time consuming and expensive to design.

This paper defines a new model that uses transformation rules to help testers define tests at the platform independent model level. The transformation rules have been applied to six large avionic applications. The results show that the new model reduced the difference between model and code with respect to the number of additional test requirements from an average of $67 \%$ to $0 \%$ in most cases and less than $1 \%$ for all applications.
\end{abstract}

\section{INTRODUCTION AND BACKGROUND}

A platform independent model is a design model that ignores concerns regarding the implementation language, operating system, and target hardware. A model compiler translates a design model to code, directly or via an intermediate platform specific model. A model compiler is free to transform a design model in any way as long as the behavior of the transformed program is the same as specified in the design model. Hence, the structure of the code might differ from the structure in the design model [1].

The fact that the structure might differ between design model and code becomes a problem when applying structural coverage criteria for testing, since full test coverage for a design model does not necessarily mean full test coverage for the code. This problem was recognized by Kirner [2], who identified four issues for determining structural code coverage at the model and code level:

(i) The modeling language may use a different implementation style (e.g., data flow instead of control flow).

(ii) The modeling language may use abstractions that hide details of the implementation, making it harder to identify structural entities to cover within the model. (iii) Code generation may be parameterizable (i.e., model semantics and implementation depend on the code generation settings).

(iv) Many modeling environments are continuously being developed, so the semantics of language constructs change over time. Further, modeling languages are rarely standardized; each tool provider often has its own modeling language.

These potential problems apply to both design models with automatic code generation and manual coding. We previously showed that the difference in the number of test requirements between the design model and the generated source code can be very large, an average of more than $60 \%$ for our subjects [3]. This paper proposes transformation rules to address this problem.

The US Federal Aviation Administration (FAA) requires full MCDC [4] code coverage for certifying safety critical avionics software with the highest safety level, and each test must be derived from the requirements. The latter is considered to be crucial for recognizing unintended or missing functionality, problems that could be missed by applying test criteria directly to the code. Hence, engineers must satisfy MCDC but cannot use the code structure code for test design, only to measure the tests. This makes the process of defining test requirements and test values time consuming and expensive. To overcome this problem, engineers need methods that allow us to apply structural coverage criteria on a design model in a way that also covers the structure of the code. This paper defines transformation rules on the model that make implicit predicates explicit, thus addressing this problem. The method has been applied to real avionic software applications. The applications are the same as we used in the previous study [3].

The rest of the current section gives the necessary background to logic-based criteria and a summary of our previous work. Section II defines the transformation rules, section III describes the experimental setting, and section IV presents the results and analysis. Section V presents related work, and conclusions and future work are found in section VI. 
TABLE I

TRUTH TABLE FOR PREDICATE $p$.

\begin{tabular}{c|ccc|c|ccc} 
Entry & $a$ & $b$ & $c$ & $p=a \vee(b \wedge c)$ & $p_{a}$ & $p_{b}$ & $p_{c}$ \\
\hline 0 & $\mathrm{~T}$ & $\mathrm{~T}$ & $\mathrm{~T}$ & $\mathrm{~T}$ & $\mathrm{~F}$ & $\mathrm{~F}$ & $\mathrm{~F}$ \\
\hline 1 & $\mathrm{~T}$ & $\mathrm{~T}$ & $\mathrm{~F}$ & $\mathrm{~T}$ & $\mathrm{~T}$ & $\mathrm{~F}$ & $\mathrm{~F}$ \\
\hline 2 & $\mathrm{~T}$ & $\mathrm{~F}$ & $\mathrm{~T}$ & $\mathrm{~T}$ & $\mathrm{~T}$ & $\mathrm{~F}$ & $\mathrm{~F}$ \\
\hline 3 & $\mathrm{~T}$ & $\mathrm{~F}$ & $\mathrm{~F}$ & $\mathrm{~T}$ & $\mathrm{~T}$ & $\mathrm{~F}$ & $\mathrm{~F}$ \\
\hline 4 & $\mathrm{~F}$ & $\mathrm{~T}$ & $\mathrm{~T}$ & $\mathrm{~T}$ & $\mathrm{~F}$ & $\mathrm{~T}$ & $\mathrm{~T}$ \\
\hline 5 & $\mathrm{~F}$ & $\mathrm{~T}$ & $\mathrm{~F}$ & $\mathrm{~F}$ & $\mathrm{~T}$ & $\mathrm{~F}$ & $\mathrm{~T}$ \\
\hline 6 & $\mathrm{~F}$ & $\mathrm{~F}$ & $\mathrm{~T}$ & $\mathrm{~F}$ & $\mathrm{~T}$ & $\mathrm{~T}$ & $\mathrm{~F}$ \\
\hline 7 & $\mathrm{~F}$ & $\mathrm{~F}$ & $\mathrm{~F}$ & $\mathrm{~F}$ & $\mathrm{~T}$ & $\mathrm{~F}$ & $\mathrm{~F}$ \\
\hline
\end{tabular}

\section{A. Logic-Based Coverage Criteria}

A predicate is an expression that evaluates to a Boolean value. Predicates consist of one or more clauses. A clause is a Boolean variable, non-Boolean variables combined by a relational operator, or a call to a Boolean function. For example, the predicate $p=(a<b \wedge D) \vee f(x)$ contains three clauses: a relational expression $(a<b)$, a Boolean variable $D$, and a Boolean function call $f(x)$. The most common logical operators in source code are negation $(\neg)$, and $(\wedge)$ and or $(\vee)$. Other logical operators, implication $(\rightarrow)$, exclusive or $(\oplus)$ and equivalence $(\leftrightarrow)$, are more common in specification languages.

Logic-based test coverage criteria focus on covering clauses (clause coverage), predicates (predicate coverage), or subsets of evaluations of predicates (e.g., active clause coverage) [5]. Ammann et al. [5] presented three different interpretations of the MCDC definition, which they defined as GACC, CACC and RACC, all variants of active clause coverage.

A clause $c$ in a predicate $p$ is considered to be active if the other clauses in $p$ have values such that changing the value of $c$ changes the value of $p$.

Definition 1: [Correlated Active Clause Coverage (CACC)] For each $p \in P$ and each major clause $c_{i} \in C_{p}$, choose minor clauses $c_{j}, j \neq i$ so that $c_{i}$ determines $p$ ( $c_{i}$ is active). Then define two requirements for each $c_{i}$ : $c_{i}$ evaluates to true and $c_{i}$ evaluates to false. The values chosen for the minor clauses $c_{j}$ must cause $p$ to be true for one value of the major clause $c_{i}$ and false for the other [5].

Consider the predicate $p=a \vee(b \wedge c)$. It has three clauses, thus we get six test requirements. Some are redundant, leaving four unique test requirements. To make $a$ active, $(b \wedge c)$ must be false while $a$ is assigned the value true once and false once. The expression $(b \wedge c)$ can be false in three ways, any of which can be used to show $a$ 's independent effect on the predicate evaluation.

Consider the truth table in Table I. The table shows the truth values for the individual clauses as well as the predicate. It also shows which clauses are active. For example $p_{a}$ is true if changing the value of $a$ would affect the value of the predicate.

Clause $a$ is made active by rows $\{1,2,3,5,6,7\}$ from table I. So CACC is satisfied by choosing one of rows 1,2 , or 3 (where $a$ is true), and pairing it with one of rows 5, 6, or 7 (where $a$ is false). Clauses $b$ and $c$ only have one pair of choices each, $\{4,6\}$ and $\{4,5\}$. Hence, the test requirements must include rows 4,5 and 6 to cover $b$ and $c$. Given that we must choose $\{4,5,6\}$, we can simply add any entry from $\{1,2,3\}$ to also cover $a$. This gives us three choices for minimal sets of rows, $\{1,4,5,6\},\{2,4,5,6\}$, or $\{3,4,5,6\}$, to satisfy CACC for predicate $p$. To achieve $100 \%$ CACC coverage given a predicate with $N$ clauses the minimal number of test requirements (TR) is $N+1$ and the maximal number is $2 N$ [5].

Predicate Coverage (PC) requires just one pair of test requirements for predicate $p, p=$ true and $p=$ false. These can be satisfied by combining any of rows $\{0,1,2,3,4\}$ with any of rows $\{5,6,7\}$. To achieve $100 \%$ PC coverage, we therefore get two test requirements per predicate. For each clause $c$, Clause Coverage (CC) requires one test requirement such that $c=$ true and one such that $c=$ false. The test requirements for CC can however, be satisfied by combining two rows with different evaluations for all clauses, i.e., $\{0,7\},\{1,6\},\{2,5\}$ or $\{3,4\}$ in the example above. The number of test requirements for a predicate is therefore two, independent of the number of clauses.

To estimate the number of test requirements for an application, we can therefore count the number of clauses and predicates and then compute the number of test requirements for different logic-based coverage criteria.

Stronger logic criteria such as CACC and MCDC are only needed when predicates have more than one clause. If a predicate has a single clause, CACC and MCDC (and CC) collapse to simply PC.

\section{B. Relationships and Associations}

Classes in a class diagram do not exist in isolation, they are interrelated. We have to formalize the knowledge of how these classes are associated through their associations in the model. A relation is an abstraction stating that instances from certain classes are associated in some way. A UML relationship covers associations, generalization, and dependencies. xtUML defines associations between classes (Figure 1), association classes that formalize associations, and generalizations (Figure 2). An xtUML association definition is in definition 2.

Definition 2: An association is the abstraction of a set of domain relationships that hold systematically between different kinds of conceptual entities, abstracted as classes, in the domain.

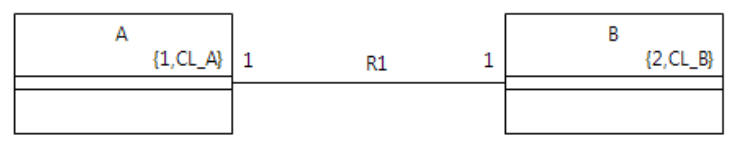

Fig. 1. Example of a binary association in UML.

The following restrictions regarding UML associations are applied to xtUML models [6]:

- Association: Generalizations are always notated as disjoint and complete. No support for implementation spe- 
TABLE II

TEST REQUIREMENTS FOR LOGIC-BASED CRITERIA.
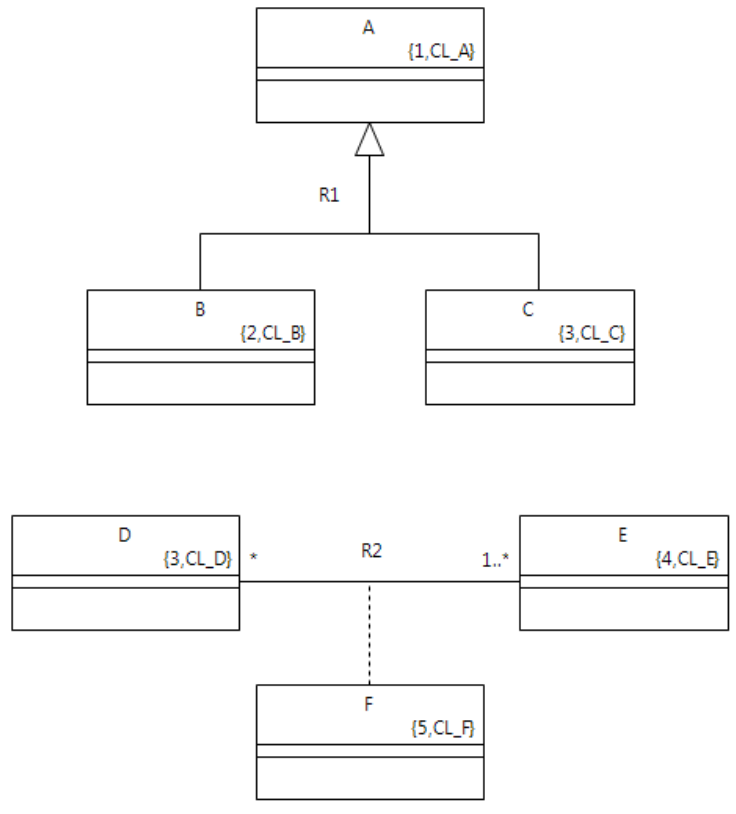

Fig. 2. Example of an association class and generalization in UML.

cific constructs, like aggregation and composition, is provided

- Association End: Associations between classes are always named, have verb phrases on both ends specifying the roles, and have multiplicity specified at both ends.

- Multiplicity: Multiplicities at association ends are restricted to 1 (exactly one), 1..* (one to many), $0 . .1$ (zero to one), and * (zero to many). The first two, the ones that start with one, are unconditional because at least one instance must participate. The last two, the ones that start with zero, are conditional.

- Navigability: The association can be navigated in both directions.

\section{Association Navigation}

The UML standard [7] defines the actions that can be used in behavioral specifications. Two kinds of actions are defined, write and read. The first can modify the system state and the second can read the system state. The defined actions specific for associations are CreateLink, DeleteLink and ReadLink. This study focuses on ReadLink. The result from ReadLink is an instance or an instance set for the navigation target.

We have used xtUML for our study but both xtUML and fUML [8] depend on the same kind of actions for reading and manipulating associations. As an example of the similarity between the actions used for xtUML and fUML, consider the example given by Mellor and Balcer [6]. They use the Online Bookstore Domain Case Study as an example, which was later

\begin{tabular}{|c|r|r|r|r|r|r|}
\hline \multirow{2}{*}{ Application } & \multicolumn{6}{|c|}{ Test Requirements, model $M$ and code } \\
\cline { 2 - 7 } & \multicolumn{1}{|c|}{ Model Level } & \multicolumn{2}{c|}{ Code Level } & \multicolumn{2}{|c|}{ Increase (\%) } \\
\cline { 2 - 7 } & $2^{*} P$ & $P+C$ & $2^{*} P$ & $P+C$ & $2^{*} P$ & $P+C$ \\
\hline A1 & 470 & 478 & 586 & 594 & 25 & 24 \\
\hline A2 & 790 & 815 & 1128 & 1153 & 43 & 41 \\
\hline A3 & 60 & 60 & 70 & 70 & 17 & 17 \\
\hline A4 & 98 & 100 & 118 & 120 & 20 & 20 \\
\hline A5 & 242 & 242 & 246 & 246 & 2 & 2 \\
\hline A6 & 2254 & 2345 & 4482 & 4579 & 99 & 95 \\
\hline \hline Total & 3914 & 4040 & 6630 & 6762 & 69 & 67 \\
\hline
\end{tabular}

adapted and used in the OMG standard for Alf [9], a definition of an action language for fUML.

\section{Previous Work}

As we showed in a recent study, the impact on the number of test requirements when transforming a platform independent design model to code can be large [3]. Six applications were used in the study. All six are aviation applications developed in xtUML by separate teams in Saab Aeronautics for the Gripen fighter ${ }^{1}$.

The number of predicates and clauses, referred to as test artifacts, were counted before and after the transformation to $\mathrm{C}++$ code. Based on the number of predicates $P$ and clauses $C$, the number of test requirements were calculated for each application for various logic-based criteria. The results showed that the increase in the number of test requirements for logicbased coverage criteria such as MCDC can be large. Table II shows statistics on the predicates and clauses from the six applications used in our study. The two Model Level columns show the number of predicates times two (each must be made true and false), and the sum of the number of predicates and clauses. As stated previously, the minimal number of test requirements is $\mathrm{N}+1$, where $\mathrm{N}$ is the number of clauses in a single predicate, so with a total of $\mathrm{C}$ clauses and $\mathrm{P}$ predicates, the program has a minimum of $\mathrm{P}+\mathrm{C}$ test requirements for MCDC. The Code Level columns show the same numbers for the $\mathrm{C}++$ code, and the Increase columns show the percent increase of the code over the model.

The increase in the minimum number of test requirements for MCDC coverage $(P+C)$ is $95 \%$ for one of the applications (A6 in Table II). This result indicates that the model contains conditions that are not expressed explicitly as predicates. Hence, by measuring the coverage by applying a logic-based criterion such as MCDC to the model we would not measure the coverage with respect to these conditions and therefore, they might not be fully covered by the tests. We call such conditions implicit predicates. In order to fully cover the semantic behavior of a design model by tests, we need to enforce both true and false evaluation of such implicit predicates. This requires a stronger criterion than MCDC at the model level.

A predicate or clause in the code that does not have a corresponding predicate or clause in the design model can

\footnotetext{
${ }^{1}$ http://saabgroup.com/Air/Gripen-Fighter-System
} 
still be traced to its source in the design model. Our previous study identified some sources for implicit predicates and also presented an initial idea for how implicit predicates can be transformed into explicit predicates for xtUML models without introducing any platform dependency [3]. This paper presents a set of rules for transforming the implicit predicates in the model to explicit predicates. Our results address issue (ii) presented by Kirner [2], where the abstraction corresponds to the sources of implicit predicates. The test artifacts introduced by the model compiler and their correspondence in the implementation need to be traceable to compare test requirements from the model to the implementation. The transformation from implicit to explicit predicates and to an implementation will depend, as mentioned in Kirners' issue (iii), on the parameters in the code generator. Hence, a general solution needs to be robust to different translations.

An analysis of the design model and the generated code shows that most new predicates come from two sources, actions that represent chained navigation of associations, and iteration over instance sets in the xtUML models. The chained navigation statements are transformed into several actions in the $\mathrm{C}++$ code, where each action handles one step in the chain. This allows a method to make implicit predicates explicit by a set of implicit to explicit transformation rules, improving our ability to cover the code structure at a platform independent level.

\section{The Model Transformation Rules}

As pointed out in Section I-D, we need to enforce both true and false evaluations of the implicit predicates. For example, an association with multiplicity zero-to-many must be exercised by at least one test with zero instances and at least one test with at least one instances.

This paper defines a set of transformation rules to solve the problem identified previously.

The basic idea is that the deterministic transformation rules are applied to a design model $M$ to generate a modified model $M^{\prime}$ '. Figure 3 shows the setup in our previous study [3], where we found that the $\mathrm{C}++$ code had more predicates and clauses than the model. Figure 4 shows the new modified process, transforming $M$ to $M^{\prime}$. Predicates and clauses are counted in $M^{\prime}$ (in Figure 4) and compared to the number of predicates and clauses from the original model $M$ and in the $\mathrm{C}++$ code generated from the original model $M$, (in Figure 3). $M^{\prime}$ is generated solely for the purpose of testing and is not used for code generation.

We generate test requirements from $M^{\prime}$ and these can be used to measure the tests on the model $M^{\prime}$ ' until full coverage is reached. These tests will then not only give full coverage of the semantic behavior of $M$, but hopefully also significantly higher MCDC coverage for the implementation than without the use of $M^{\prime}$. As mentioned earlier the tests must be derived from the requirements and not from the design model structure. How high the coverage is for the implementation depends on the success of the transformation rules with respect to catching all the sources for implicit predicates. An initial evaluation is presented in section III. The smaller the difference is between $M^{\prime}$ and the code with respect to predicates and clauses, the more useful is this approach in supporting test design for safety critical systems.

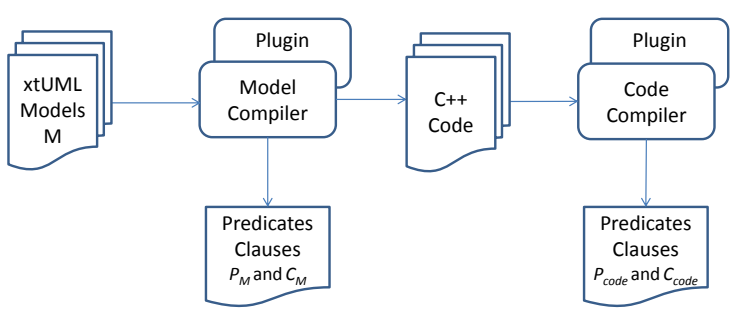

Fig. 3. A framework that counts all predicates and clauses in a model $M$ and in the generated code. There is an increase in the code compared to $M$.

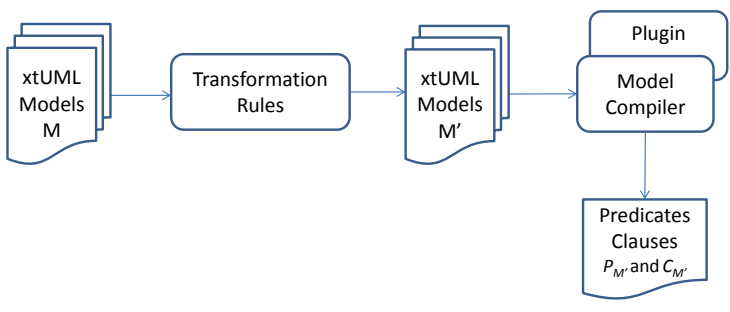

Fig. 4. The framework as the transformation rules are applied. The number of predicates and clauses in $M$ 'is compared to the code generated from the original model $M$.

\section{A. Implicit to Explicit Transformation Rules}

We have identified three sources for implicit predicates, and used them to define a set of transformation rules. The application of a transformation rule to a source of an implicit predicate in the model adds an explicit predicate with one clause to that part of the model. This is done in the object action language (OAL) without introducing any platform dependencies. Transformation rules on three constructs are described below.

1) For-Each Statement (FEACH):

The for each statement is an iterator that operates over a set of instances. The <statements $>$ in the loop are executed against each <instance> in the <instance set>. syntax:

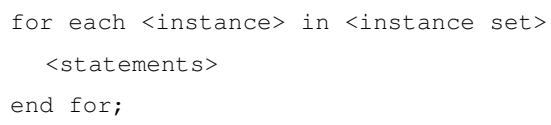

The example below iterates over an instance set If_Stmts that represents a snapshot of the current class extent for the class that represents an if statement in the meta-model for OAL, and has the unique identifier ACT_IF.

Original OAL:

select many If_Stmts instances of ACT_IF; 
for each If_Stmt in If_Stmts

<statements>

end for;

There is no explicit exit criterion in the OAL for the for each statement. We can interpret that by adding an if statement as a guard around the for each statement.

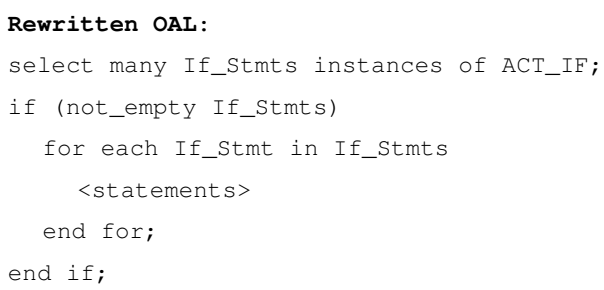

This transformation rule ensures iterator coverage for the for each statement, where iterator coverage in this case should be interpreted as having at least one test execution with one or more iterations and (if possible) at least one test execution with zero iterations. MCDC would ensure this at code level but not necessarily at model level.

2) Association Navigation (ANAV): Before introducing the set of transformation rules for association navigation we will look at the multiplicity of an association class and the generalization. Figure 2 has no explicit multiplicity for a navigation with start from the association class to the other participants. The same is true for the generalization, which is independent of navigation direction. We can illustrate this by doing a conceptual transformation of the relationships, as shown in Figure 5. The multiplicity when navigating from a super type class to a sub-type class is $0 . .1$. The reason is that a super type can only be associated with one sub-type instance. If an instance of a sub-type class exists, a super type class must also exist. This example illustrates one of the restrictions disjoint and complete mentioned in I-B. If an instance of an association class exists, this implies also that instances of the associated classes exist, therefore the multiplicity is 1 .

The select statement in the object action language OAL is used to assign an instance or instance set to a local variable with a data type that represents the assigned value. The following applies:

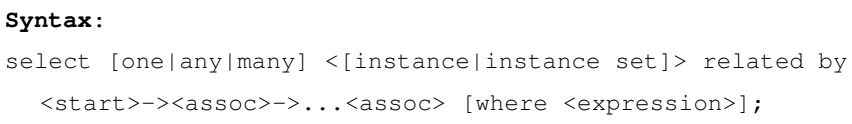

where:

- select one is used if at most one instance can be returned by navigating the associations.

- select any or select many is used if more than one instance can be returned by navigating the associations. select any returns a single instance and select many returns all instances at the end of the navigation.

- If any of the relationships are conditional in the direction of navigation the returned instance or instance set can be empty.
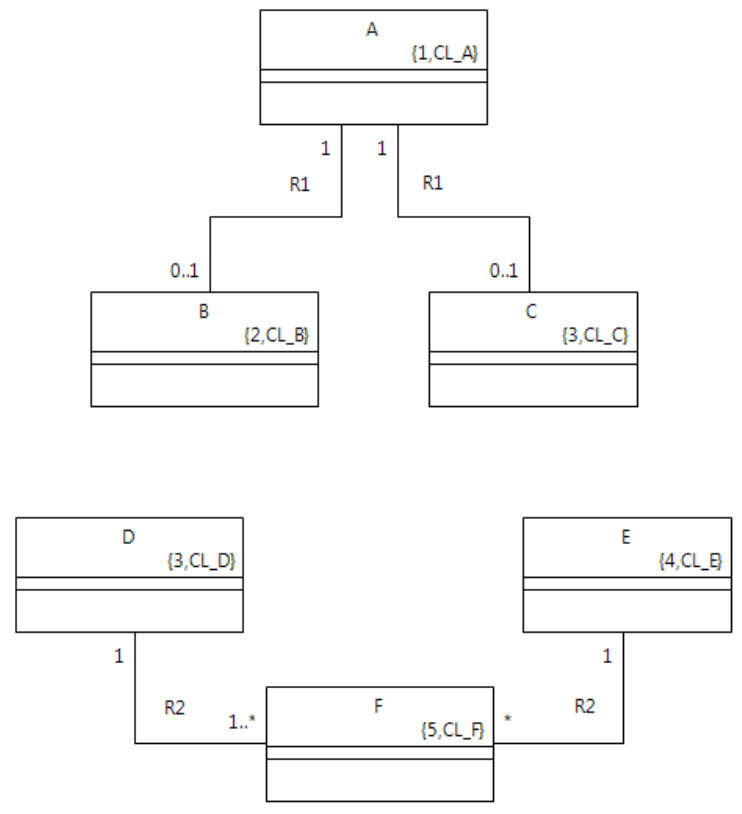

Fig. 5. Conceptual transformation of the associations in Figure 2.

- <start> is an instance or an instance set and is not allowed to be empty.

- The expression $-><$ assoc $>$ corresponds to a step in the navigation, where $->$ is literal and $\langle$ assoc $\rangle$ is the specification of the relationship from the source to the destination class. The association is obtained by combining the unique class identifier and the unique association name, e.g., V_VAL [R625], where V_VAL is the class identifier, and [R625] is the association name within literal square brackets.

- [where <expression>] is optional and specifies a selection criterion that is applied at the end of the navigation. The resultant instance or instance set fulfills the selection criterion at the end of the navigation.

We substitute each step $<$ start $>-><$ as soc $>->$... with the correspondent multiplicity $<\mathrm{m}>$ for each navigation end, for example $<$ start $>-><m>->\ldots$. Due to the restriction that <start> cannot be empty, we have only two possible values for $\langle m\rangle,\langle 1\rangle$ and $\langle 1 \ldots *\rangle$. This is because each step is handled separately and the intermediate step result is assigned to a temporally local variable $\langle$ class_id_instance $(s)\rangle$, which serves as start variable for next navigation step. The start variable for the first navigation step is explicitly specified in the select statement as <start>.

A depth first navigation approach is used, meaning that the for each statement is used when the start for a navigation step is an instance set. The reason for the choice is that it matches the rules when a selection criterion exists. 
The breadth first approach could also have been used; the transformation rules are independent of navigation approach. The important semantic we want to capture is embedded in the conditional navigation steps. After the substitution the navigation will be executed iteratively and the following rules can be applied to each step individually. To increase readability of the rules below, the original OAL is presented in bold while the rewritten OAL is written in script.

The first two rules apply during the navigation as long as steps remain. These are rules for next step, where the start variable has multiplicity $-><m>->$ in the navigation.

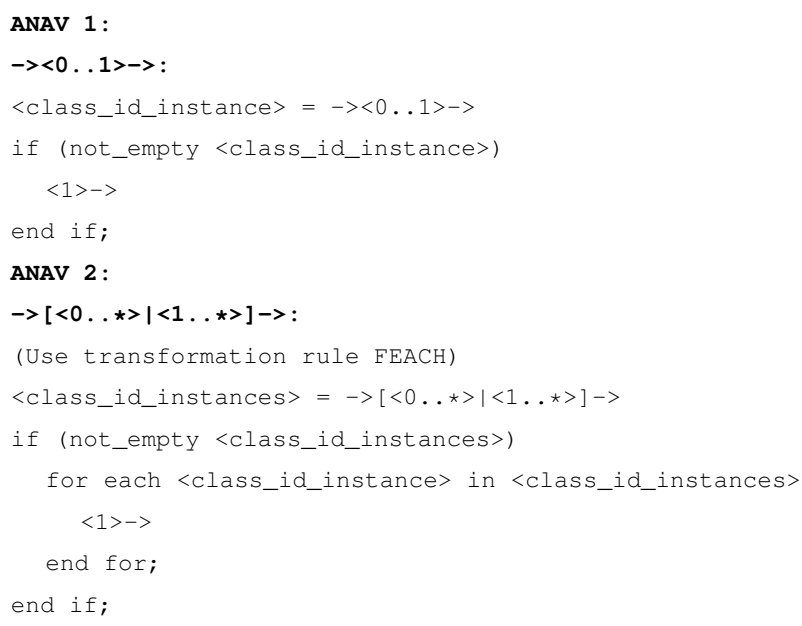

The rules ensure that there will be at least one test with no instance and at least one test with at least one instance. As the rules are applied at each step, we can capture the conditional behavior of a chained navigation. At the end of a chained navigation, the rules differ because we need to handle the result (select any or many). We get different rules depending on (i) the cardinality of the select statement, and (ii) whether there is a selection criterion, that is, a where <expression>. We begin with two rules for a select any <instance> ... where <expression> statement.

ANAV 3:

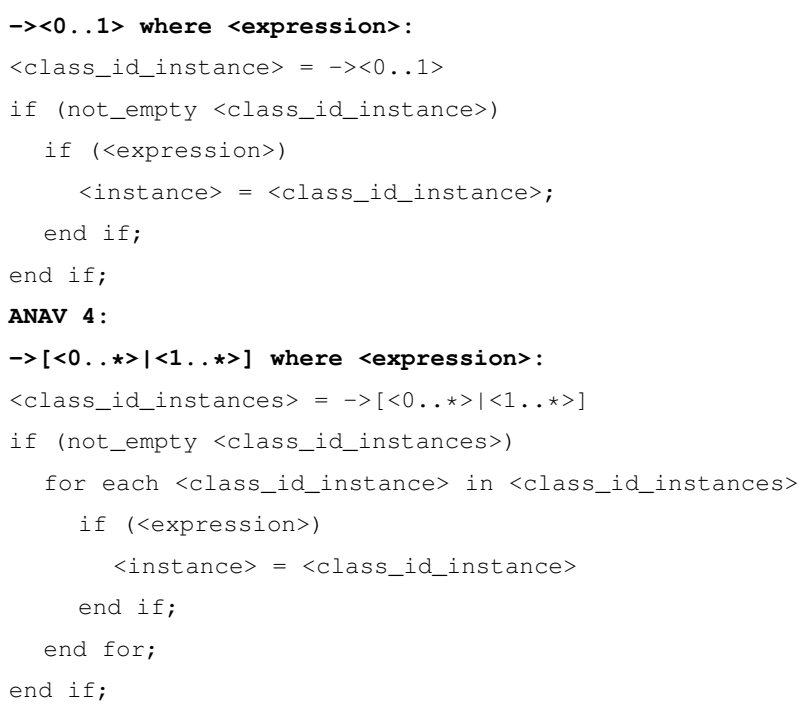

The following two rules are applied on select many <instances> ...where <expression> statements.

\section{ANAV 5:}

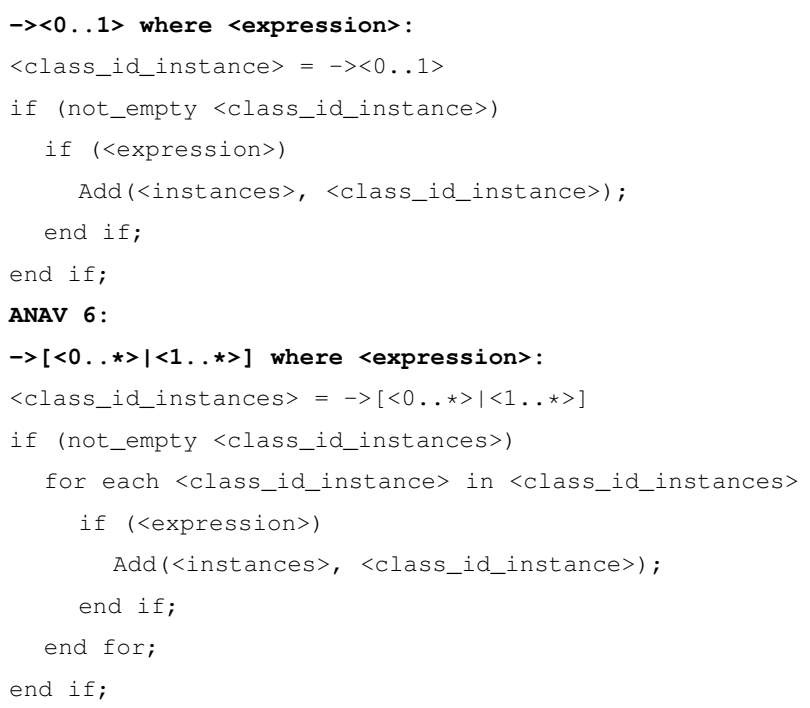

The following is a rule for a select any <instance> ... statement without selection criterion.

ANAV 7:

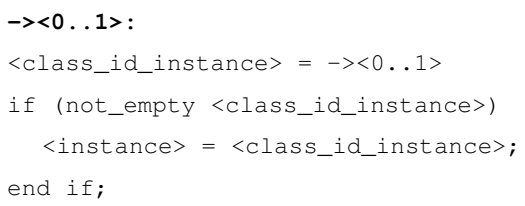

Finally, we have two rules for a select many <instances $>$... statement without selection criterion.

ANAV 8:

$-><0$. .1>:

$<$ class_id_instance $>=-><0.1\rangle$

if (not_empty <class_id_instance>)

Add (<instances>, <class_id_instance>);

end if;

\section{ANAV 9:}

$\rightarrow<0 \ldots *>$ :

$\langle$ class_id_instances $>=->\langle 0 \ldots *\rangle$

if (not_empty <class_id_instances $>$ )

AddAll (<instances>, <class_id_instances>); end if;

Example: The OAL statement below will select all instances of the super type class ACT_SMT that are also a subtype of the class $A C T \_I F$, and assign the result to the local instance set variable If_Stmts, by navigating the association R603 within the meta-model for the OAL.

Original OAL:

select many If_Stmts related by Stmts->ACT_IF[R603];

When substituted, the statement above yields $\langle 1 \ldots *\rangle-\rangle\langle 0 \ldots 1\rangle$ and we can see that ANAV 2 applies to the first step, $\langle 1 \ldots \star>->$, and that ANAV 8 applies to the final step since we have select many, no condition and $-><0 \ldots 1>$.

After applying the transformation rules ANAV 2 for the first step and ANAV 8 to the last step we get the following OAL. Rewritten OAL :

if (not_empty Stmts) 


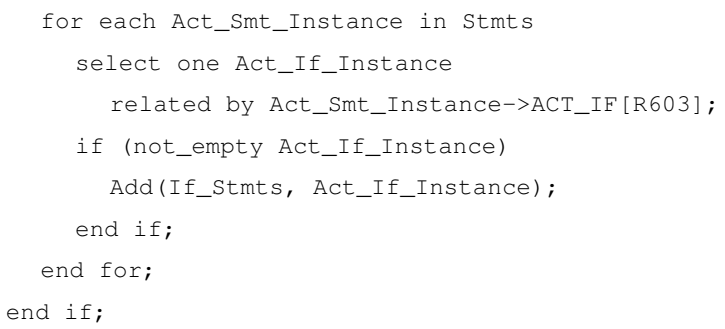

The ANAV rules ensure all-navigation coverage for class associations, where all-navigation coverage should be interpreted as each conditional link in a chained navigation is evaluated to empty at least once and not empty at least once in the direction of the navigation. MCDC does not ensure this at the model level.

3) Derived Attribute (DATTR):

An attribute is derived if its value can be computed from other attributes already in the model. Derived attribute dependencies can span multiple classes. In xtUML, writing to a derived attribute is not allowed and the model compiler is responsible for guaranteeing the constraint before the attribute can be read. The constraint is written in OAL and must be assigned a value.

Original OAL:

self.IsHalfFull = self.Volume $>$

$\left(\Pi \star\right.$ self.Radius $^{2}$ * self.Height) $/ 2$;

We can interpret the attribute read as a function call that returns the attribute value. The function behavior is the original constraint combined with a return statement. The rule is only applied to derived attributes with data type Boolean. These introduce conditions and thereby affects predicates and clauses.

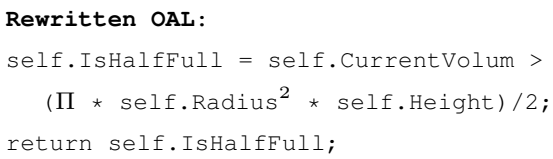

This transformation rule ensures that conditions introduced by derived attributes are evaluated to true at least once and false at least once. MCDC would ensure this at code level but not necessarily at the model level.

\section{THE STUDY}

This section evaluates the impact that the implicit to explicit transformation rules have on test artifacts, i.e., predicates and clauses. The experiment is quantitative and assesses and compares the number of predicates and clauses at the model level before and after the implicit to explicit transformation rules are applied. These are also compared with the number of predicates and clauses at the code level. Figures 3 and 4 illustrate the framework for this experiment.

\section{A. Subjects}

The empirical study uses the aviation system software developed in xtUML by Saab Aeronautics that was previously studied [3]. Saab Aeronautics is responsible for development, production, marketing, selling and supporting of the Gripen fighter. Our subjects are six aviation applications for an updated version of the Gripen, developed by separate teams.
Table III summarizes the six applications. The columns under "Action Homes" represent places in the application where the behavior is expressed in the object action language (OAL), using states and operations. One application, A6, is actually a part of the model compiler itself, the functionality that transforms the OAL constructs. This part of the model compiler is modeled in xtUML and transformed into $\mathrm{C}++$.

TABLE III

SUMMARY OF EXPERIMENTAL SUBJECTS

\begin{tabular}{|c|c|c|c|c|c|}
\hline & & & \multicolumn{4}{|c|}{ Action Homes } \\
\cline { 4 - 6 } Applications & Components & \multirow{2}{*}{ Classes } & States & Operations & Others \\
\hline A1 & 9 & 61 & 117 & 133 & 60 \\
\hline A2 & 7 & 66 & 117 & 137 & 79 \\
\hline A3 & 3 & 12 & 15 & 29 & 6 \\
\hline A4 & 3 & 18 & 15 & 27 & 7 \\
\hline A5 & 3 & 36 & 81 & 112 & 28 \\
\hline A6 & 4 & 444 & 2 & 582 & 51 \\
\hline \hline Total & 29 & 637 & 347 & 1020 & 231 \\
\hline
\end{tabular}

\section{B. Tools and procedure}

This experiment used two tools, the BridgePoint ${ }^{2}$ product for the xtUML modeling environment and model compiler, and the compiler framework $\mathrm{LLVM}^{3}$ for the $\mathrm{C}++$ code analysis. These tools do not directly support our needs for data collection, so we developed plugins to extend the functionality. The model compiler used with BridgePoint is designed and developed within Saab Aeronautics, where the first author is a developer. The same configuration of the model compiler is used for all six applications.

As mentioned, the data collection functionality is designed and implemented into BridgePoint and LLVM as plugins. Both plugins count all predicates and clauses, emitting their results as plain text files, as in Figure 3. The plugin for BridgePoint traverses the xtUML meta-model (populated with instances from the applications) for data collection. The plugin for LLVM traverses the in-memory abstract syntax tree (populated with instances from the generated $\mathrm{C}++$ applications) for data collection.

One important feature of the model compiler is that for every $\mathrm{C}++$ function generated, a label with a unique identifier derived from the corresponding $x t U M L$ model element is emitted as the first statement in the outermost block of that function. By recognizing the label as an ordinary $\mathrm{C}++$ label statement during data collection, we can establish traceability to a certain degree between the code level and the model level.

The new implicit to explicit transformation rules defined in II-A were implemented and added into the plugin for BridgePoint, as in Figure 4. Functionality to measure the effect of each transformation rule to the counting result was also included.

\section{RESUlts}

Table II showed the number of test requirements after applying the logic-based criterion such as MCDC to the

\footnotetext{
${ }^{2}$ http://www.mentor.com/products/sm

${ }^{3}$ http://llvm.org
} 
original design model $M$ and to the generated $\mathrm{C}++$ code for the six applications. These are the results from our previous study where we did not use the implicit to explicit transformation rules. Table IV shows the number of test requirements for the same applications after applying the implicit to explicit transformation rules to the original design models to create $M^{\prime}$. The MCDC test requirements for $M^{\prime}$ therefore covers the original model $M$ not only with respect to MCDC but also with respect to all-navigations. For four applications, the number of additional test requirements drops to zero, indicating that the predicates and clauses in the transformed design model are the same as in the generated $\mathrm{C}++$ code. Thanks to the unique labels, we can verify that the number of predicates and clauses in each $\mathrm{C}++$ function is the same as in its corresponding xtUML element. For two of the applications, the number is slightly above zero, indicating that there are some additional sources to implicit predicates that our method does not cover. A further analysis has been done to understand these additional implicit predicates.

Table $\mathrm{V}$ shows the distribution of the sources to implicit predicates and clauses that our method considers, ANAV, FEACH and DATTR. We can see that by just applying FEACH and ANAV, most of the implicit predicates became explicit. DATTR was only applied four times.

TABLE IV

TEST REQUIREMENTS FOR LOGIC-BASED CRITERIA AFTER TRANSFORMATION

\begin{tabular}{|c|r|r|r|r|r|r|}
\hline \multirow{3}{*}{ Applications } & \multicolumn{6}{|c|}{ Test Requirements, model $M^{\prime}$ and code } \\
\cline { 2 - 7 } & \multicolumn{1}{|c|}{ Model Level } & \multicolumn{2}{c|}{ Code Level } & \multicolumn{2}{|c|}{ Increase $(\%)$} \\
\cline { 2 - 7 } & $2 * P$ & $P+C$ & $2 * P$ & $P+C$ & $2 * P$ & $P+C$ \\
\hline A1 & 582 & 590 & 586 & 594 & 0.7 & 0.7 \\
\hline A2 & 1128 & 1128 & 1128 & 1153 & 0 & 0 \\
\hline A3 & 70 & 70 & 70 & 70 & 0 & 0 \\
\hline A4 & 118 & 120 & 118 & 120 & 0 & 0 \\
\hline A5 & 246 & 246 & 246 & 246 & 0 & 0 \\
\hline A6 & 4472 & 4573 & 4482 & 4579 & 0.2 & 0.1 \\
\hline \hline Total & 6616 & 6727 & 6630 & 6762 & 0.2 & 0.5 \\
\hline
\end{tabular}

TABLE V

SUMMARY OF IMPLICIT TRANSFORMATION RULE CONTRIBUTIONS.

\begin{tabular}{|c|r|r|r|}
\hline \multirow{2}{*}{ Applications } & \multicolumn{3}{|c|}{ Implicit to Explicit Transformation Rules } \\
\cline { 2 - 4 } & \multicolumn{1}{|c|}{ ANAV } & \multicolumn{1}{|c|}{ FEACH } & DATTR \\
\hline A1 & 11 & 35 & 0 \\
\hline A2 & 63 & 105 & 1 \\
\hline A3 & 3 & 2 & 0 \\
\hline A4 & 6 & 3 & 1 \\
\hline A5 & 0 & 2 & 0 \\
\hline A6 & 720 & 387 & 2 \\
\hline \hline Total & 804 & 584 & 4 \\
\hline
\end{tabular}

\section{A. Other Sources of Predicates and Clauses}

We identified three other sources that account for the differences in predicates and clauses in this study.

1) Constant Expression:

The select statement shown below, with one explicit predicate, is transformed into $\mathrm{C}++$ code with no predicate. This statement can be used in two situations in OAL. The first is to assign an empty instance or instance set to a local variable, and the second is to explicitly declare a local variable with a specific scope. A local variable in OAL is implicitly declared as soon as it is given a value, with the scope limited to the current block.

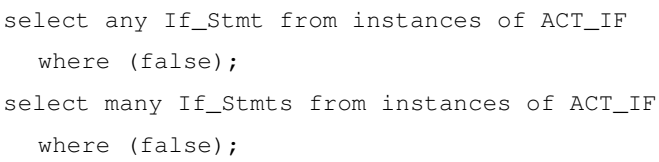

This source for implicit predicates is extra clauses in the exit criteria for the for loop used in the $\mathrm{C}++$ code. This happens when specifying an association navigation statement select any that has more then one association-end with multiplicity $\langle 0 \ldots *\rangle$ or $\langle 1 \ldots *\rangle$ in the direction of navigation. An extra exit-variable was introduced in the $\mathrm{C}++$ code to make it possible to break all loops immediately when an instance is found. This is also valid when a selection criterion where <expression> exists.

3) Signal Delegation:

This case has to do with ports in a component. Each port has its own procedure where the OAL can be used, but if the signal is delegated to a class-based state machine, the specified behavior will be substituted with code generated from the model compiler. The missing predicates correspond to the assignments of a local variable with the formal parameters of the signal as values before further delegation. Procedures that represent delegated signals were not excluded from the counting at the model level, even if they do not include any OAL statements.

\section{B. Discussion of Validity}

Independent variables in this experiment are the six aviation applications specified in xtUML and the set of transformation rules. The dependent variables of the experiment are the predicates and clauses generated for the transformed models and the implementation. One possible confounding variable in this experiment can be the design guide that is followed when specifying xtUML models according to the company's development process. The design guide includes restrictions for model constructs allowed in the xtUML models. Before the xtUML models are translated into $\mathrm{C}++$ code, system design decisions are specified as markings in the model to the model compiler. These markings reflect the underlying platform; most are about partitioning and scheduling of applications on the platform and should therefore not affect the number of predicates and clauses in the $\mathrm{C}++$ implementation.

Only one model compiler was used for the transformation, thus the results might not be valid for other transformations. However, the idea regarding model-to-model transformation making the implicit predicates explicit is applicable to any application specified as an xtUML model.

This study compared the numbers of test artifacts (predicates and clauses) between models and code. Having the same number of test artifacts, of course, does not necessarily imply they are the same artifacts. We evaluated this with function 
level traceability. Specifically, we traced the test artifacts in the source code back to the corresponding xtUML elements (the "Action Homes" in Table III). This allowed us to verify that the test artifacts are indeed the same.

Finally, the method was applied to a set of real industrial applications, developed by separate teams, but all within the aerospace domain. However, the sources to implicit predicates that we identified (e.g., multiplicity of an association in a class diagram) are typical for any xtUML model, although the number of occurrences can vary. It is however, still possible that we might find additional sources for implicit predicates in other applications or other domains. This is not important in the long run for two reasons: $(i)$ the improvement with respect to code coverage will still mean significantly less effort spent on test design even if the code coverage is less than $100 \%$, and (ii) the approach we use to make implicit predicates explicit can probably be used for other sources as well.

\section{RELATED WORK}

Our method has similarities to the approach behind testability transformation described by Harman et al. [10], which is a source to source transformation. The transformed program is used by a test data generator to improve its ability to generate test data for the original program. The same idea was used by Friske and Schlingloff on state machines. The authors instrumented conditions of the state machines transitions to simulate All-Transition-Pairs with MCDC [11]. These transformations serves the same purpose as our implicit to explicit transformations rules.

Rajan et al. [12] presented an empirical study that measured the effect of program and model structures on MCDC test adequacy coverage. The study used six realistic systems from the civil avionics domain. The purpose of the study was to measure structural coverage on two versions of the implementation, with and without expression folding (inlined and non-inlined). The authors generated test suites that satisfied MCDC over a non-inlined implementation and then showed that these test suites did not satisfy MCDC for the inlined implementation. The average reduction in coverage was $29.5 \%$, primarily because the non-inlined versions did not account for the effect of masking [13]. While Rajan et al. [12] studied the effect on MCDC by different implementations, we study the effect on the test artifacts, i.e., predicates and clauses, by the model transformation.

One approach to handle structural coverage analysis at different design levels was used by Kirner [2]. This research focuses on ensuring that the structural code coverage achieved at a higher abstraction level is preserved during transformation down to lower levels. To guarantee preservation of structural coverage, Kirner defined formal properties that have to be fulfilled during the transformation between different program representations. These formal properties have been used to automatically create coverage profiles that can extend compilers to preserve any given code coverage criteria by enabling only those code optimizations that preserve it [14]. While Kirner and Haas [14] discussed coverage preservation by constraining the transformation, we define a method to identify test requirements in an xtUML model in such way that it corresponds sufficiently well to the MCDC test requirements for the source (average over 99\% in our study).

Little work has been done in the area of analyzing fUML models. Lazar et al. [15] make the analysis of fUML models possible, by the defined framework ComDeValCo (Component Definition, Validation, and Composition). The framework includes functionality for specifying components structure and behavior. The behavior is specified by using an action language defined by the authors. Later, the framework was extended by Lazar [16] to use the action language Alf. This framework might serve as a validation platform for model transformations and structural coverage at a platform independent level for our implicit to explicit transformation rules.

\section{CONCLUSiOnS AND Future Work}

This paper presents two results. First is a set of transformation rules that transform predicates that are implicit in a design model to explicit predicates in the model. These rules were developed from prior work that found that implementations contained more predicates than design models during modelbased software development. Further, the ANAV rules will not only significantly increase the MCDC coverage at the code level but also give full all-navigations coverage at the model level. The purpose of the model criterion all-navigations is to ensure that all conditional links in a chained navigation are evaluated to both empty and not empty in the direction of navigation. This will guarantee that we have tested at least two possible instantiations of runtime-objects satisfying each conditional link in the chained navigation. A more elaborate description and definition of this criterion is left for future work.

The second result is from an experiment on large industrial software applications in the aerospace domain that found the transformation rules can identify most of the implicit predicates. This allows structural tests to be designed at a platform independent model level, and still get very high coverage on the source code. This issue is important because it is much more cost-effective to design tests at the model level than at the code level. These results can lead to better model-based testing, cheaper.

The difference between model and code with respect to test artifacts, predicates and clauses, and test requirements were presented. Such test artifacts are used in structural coverage criteria for safety-critical software, in particular, MCDC. MCDC code coverage is required by the US Federal Aviation Administration (FAA) for certifying safety critical parts of the avionics software in commercial aircraft [4]. Our results show that the proposed method managed to reduce the difference between model and code with respect to these test artifacts to zero in most cases and less than one percent for all applications. This indicates that the set of rules for modelto-model transformation can help test design.

Even though the difference between model and code for two of the six applications did not drop to zero, these results 
are still very promising. A method that can identify $99.5 \%$ of all test requirement for MCDC code coverage at a platform independent level promises a significant improvement when it comes to the effort spent on test design. The implicit to explicit transformation rules from section II are deterministic and can therefore be applied by a model-to-model compiler. Moreover, even though we have only used xtUML in our study, the method is intended to be easy to adopt to ALF, the action language used for fUML [9], [8]. The OAL constructs we have focused on are very similar to ALF. Showing how this can be done is left for future work.

Many model-based testing techniques do not deal with actions in behavioral specifications. These actions specifies the fine-grained behavior of all behavioral elements in the model. The main effect of including actions in behavior specifications is that runtime-objects, for example links, are created and deleted during runtime, which makes the overall system state change continuously over time. This experimental study has helped show this by highlighting the implicit predicates included in association navigation (sequences of ReadLink actions) expressed in OAL code. This result can be interpreted to mean there is no benefit in measuring coverage at the code level before all explicit and implicit predicates are covered at the model level. The implicit predicates express the dynamic semantics specified in the behavioral specifications, with actions following the constrained rules specified as static semantics in the class diagram, meaning it is platform independent.

By applying data-flow and control-flow analysis to the behavioral specifications, including the actions, further semantics could be checked. For example, to use a ReadLink action for an association, there should exist a corresponding Createlink action for the same association. The places where the ReadLink actions for that particular association exists should be highlighted to the model designer as an incomplete or inconsistent design. Another example is that for each select any statement, there should exist a corresponding predicate that checks the returned instance so it is not empty before further access. If the implementation uses a reference or pointer to represent the instance, this can be seen as checking for a null pointer exception. To detect these kinds of incomplete or inconsistent semantics in the design mode, simulation is not required. Instead, this can be done continuously during design time, which can be more cost effective.

\section{A. Future Work}

In the future, we would like to extend our method to include traceability functionality of predicates and clauses. The traceability functionality should be platform independent and make it possible to compare test requirements derived from the model level and the code level. A further extension to our method would be to adapt the implicit to explicit transformations rules to be directly defined in the subset of Action Semantics that is valid for fUML. The data-flow and control-flow analysis discussed in section VI should also be further investigated together with the method defined by Planas et al. [17]. This may give ideas for new transformations rules, depending on the results and findings.

We plan to implement/integrate our extended method into an MDD environment and validate by measuring and comparing the coverage at the model level with the code level, before and after applying our method.

\section{ACKNOWLEDGMENT}

This work was supported in part by Research Grant 201001252 from the VINNOVA (Swedish Governmental Agency for Innovation Systems).

\section{REFERENCES}

[1] S. S. Muchnick, Advanced Compiler Design and Implementation. San Diego: Academic Press, ISBN 1-55860-320-4, 1997.

[2] R. Kirner, "Towards preserving model coverage and structural code coverage," EURASIP Journal on Embedded Systems, vol. 2009, pp. 6:16:16, January 2009.

[3] A. Eriksson, B. Lindström, S. F. Andler, and J. Offutt, "Model Transformation Impact on Test Artifacts: An Empirical Study," in Proceedings of the 9th workshop on Model-Driven Engineering, Verification and Validation, September 2012.

[4] RTCA, "RTCA Inc. DO-178B: Software Considerations In Airborne Systems and Equipment Certification. Requirements and Technical Concepts for Aviation." 1992.

[5] P. Ammann, J. Offutt, and H. Huang, "Coverage criteria for logic expression," in Proceedings of the 14th International Symposium on Software Reliability Engineering. IEEE Computer Society Press, November 2003, pp. 99-107.

[6] S. J. Mellor and M. J. Balcer, Executable UML: A Foundation for Model Driven Architecture. Boston: Addison Wesley, ISBN 0-201-74804-5, 2002.

[7] OMG, "Unified Modeling Language (UML), Superstructure, version 2.3," 2010, retrieved September 14, 2011 from http://www.omg.org/spec/UML/2.3.

[8] OMG, "Foundational Subset of Executable UML (FUML), version 1.0," 2011, retrieved September 14, 2011. http://www.omg.org/spec/FUML/1.0/.

[9] OMG, "Action Language for Foundational UML (ALF), version 1.0 - beta1," 2010, retrieved September 14, 2011 http://www.omg.org/spec/ALF/1.0/Beta1/.

[10] M. Harman, L. Hu, R. Hierons, J. Wegener, H. Sthamer, A. Baresel, and M. Roper, "Testability transformation," Software Engineering, IEEE Transactions on, vol. 30, no. 1, pp. 3 - 16, jan. 2004.

[11] M. Friske and B.-H. Schlingloff, "Improving Test Coverage for UML State Machines Using Transition Instrumentation," in SAFECOMP'07, ser. LNCS. Springer Berlin / Heidelberg, 2007, vol. 4680, pp. 301-314.

[12] A. Rajan, M. W. Whalen, and M. P. Heimdahl, "The Effect of Program and Model Structure on MC/DC Test Adequacy Coverage," in ICSE '08. New York, NY, USA: ACM, 2008, pp. 161-170.

[13] J. J. Chilenski, "An investigation of three forms of the modified condition decision coverage (MCDC) criterion," Office of Aviation Research, Tech. Rep., 2001.

[14] R. Kirner and W. Haas, "Automatic calculation of coverage profiles for coverage-based testing," 2009, Vienna University of Technology, Institute of Computer Engineering, Vienna, Austria, http://www.vmars.tuwien.ac.at/ raimund/papers/rr-2009077_kps09_coverage_profiles.pdf, last access January 2013.

[15] C.-L. Lazar, I. Lazar, B. Parv, S. Motogna, and I.-G. Czibula, "Using a fUML Action Language to Construct UML Models," in Symbolic and Numeric Algorithms for Scientific Computing (SYNASC), 2009 11th International Symposium on, sept. 2009, pp. $93-101$.

[16] C.-L. Lazar, "Integrating Alf Editor with Eclipse UML Editiors," Studia Univ. BabesBolyai, Informatica, vol. LVI, no. 3, pp. 27 - 32, sep. 2011.

[17] E. Planas, J. Cabot, and C. Gomez, "Verifying Action Semantics Specifications in UML Behavioral Models," in Advanced Information Systems Engineering, ser. LNCS. Springer Berlin / Heidelberg, 2009, vol. 5565 , pp. $125-140$ 